\title{
Thermal Conduction in Clusters of Galaxies
}

\section{Citation}

Narayan, Ramesh, and Mikhail V. Medvedev. 2001. "Thermal Conduction in Clusters of Galaxies." The Astrophysical Journal 562 (2): L129-32. https://doi.org/10.1086/338325.

\section{Permanent link}

http://nrs.harvard.edu/urn-3:HUL.InstRepos:41384940

\section{Terms of Use}

This article was downloaded from Harvard University's DASH repository, and is made available under the terms and conditions applicable to Other Posted Material, as set forth at http:// nrs.harvard.edu/urn-3:HUL.InstRepos:dash.current.terms-of-use\#LAA

\section{Share Your Story}

The Harvard community has made this article openly available.

Please share how this access benefits you. Submit a story.

Accessibility 


\title{
THERMAL CONDUCTION IN CLUSTERS OF GALAXIES
}

\author{
RAMESh NARAYAN $^{a}$ AND Mikhail V. MEdVEDEV ${ }^{b}$ \\ Draft version October 30, 2018
}

\begin{abstract}
We estimate the thermal conductivity of a weakly collisional magnetized plasma with chaotic magnetic field fluctuations. When the fluctuation spectrum extends over two or more decades in wave-vector, we find that thermal conduction is very efficient; the conduction coefficient is only a factor $\sim 5$ below the classical Spitzer estimate. We suggest that conduction could play a significant role in cooling flows in clusters of galaxies.
\end{abstract}

Subject headings: galaxies: clusters: general — cooling flows — conduction — magnetic fields

\section{INTRODUCTION}

Hot X-ray-emitting gas is ubiquitous in clusters of galaxies (Sarazin 1988). Since the X-ray emission is energetically important in many clusters, it is believed that a significant amount of mass must continuously cool and drop out of the intracluster medium (Fabian 1994). The mass deposition rate is estimated to be as much as several hundred $M_{\odot} \mathrm{yr}^{-1}$ in some clusters (e.g., David et al. 2001; Allen et al. 2001).

Direct evidence for the cooling gas has, however, been scarce (Fabian 1994). In particular, recent observations with XMM-Newton (Böhringer et al. 2001: Molendi \& Pizzolato 2001) and Chandra (Fabian et al. 2001) have failed to find the multi-temperature gas one expects in a cooling flow. The observations suggest that mass dropout may be less significant than previously thought. A reduced level of mass dropout is possible if there is a source of heat to replace the energy that is lost through X-ray emission, but no clear heat source has yet been identified (Fabian et al. 2001).

The inner region of a cluster $(R \sim$ few $\times 10 \mathrm{kpc})$ where mass dropout appears to be occurring is typically cooler than the rest of the cluster. Therefore, an often-discussed source of heat is thermal conduction from the hot outer regions of the cluster to the center (Binney \& Cowie 1981; Tucker \& Rosner 1983; Bertschinger \& Meiksin 1986; Bregman \& David 1988; Gaetz 1989; Rosner \& Tucker 1989; Pistinner \& Shaviv 1996; Santos 2001). While the idea is attractive, it requires extremely efficient conduction, which is considered problematic.

In a classic paper, Spitzer (1962) showed that thermal conduction in an unmagnetized plasma has a diffusion con$\operatorname{stant}^{3} \kappa_{\mathrm{Sp}} \sim \lambda^{2} / t_{\text {Coul }}=\lambda v_{t} \sim 4 \times 10^{32} T_{1}^{5 / 2} n_{-3}^{-1} \mathrm{~cm}^{2} \mathrm{~s}^{-1}$, where $t_{\text {Coul }}=\lambda / v_{t}$ is the mean free time between Coulomb collisions, and $\lambda$ and $v_{t}$ are the mean free path and the thermal speed of electrons Cowie \& McKee 1977; Ettori \& Fabian 2000): $\lambda \sim 30 T_{1}^{2} n_{-3}^{-1} \mathrm{kpc}, v_{t} \sim\left(k T / m_{e}\right)^{1 / 2} \sim$ $4 \times 10^{9} T_{1}^{1 / 2} \mathrm{~cm} \mathrm{~s}^{-1}$. Here, $T_{1}=k T / 10 \mathrm{keV}$ is the scaled temperature, $n_{-3}=n / 10^{-3} \mathrm{~cm}^{-3}$ is the scaled electron number density, and we have used an average value for the Coulomb logarithm, $\ln \Lambda \sim 38$.

The time required for heat to diffuse conductively across a radius $R=100 R_{2} \mathrm{kpc}$ is given by $t_{\mathrm{Sp}} \sim R^{2} / \kappa_{\mathrm{Sp}} \sim$ $8 \times 10^{6} T_{1}^{-5 / 2} n_{-3} R_{2}^{2}$ yr. For conduction to have a significant effect on a cooling flow, the conduction time must be comparable to the cooling time $t_{\text {cool }}$ of the gas. Table 1 lists representative data for two clusters, Hydra A (David et al. 2001) and 3C295 (Allen et al. 2001), at two characteristic radii, $100 \mathrm{kpc}$ and $10 \mathrm{kpc}$. Columns 6 and 7 give $t_{\text {cool }}$ and $t_{\mathrm{Sp}}$. We see that if thermal conduction in a cluster is as efficient as in Spitzer's theory, or even if it is a factor of a few less efficient, heat conduction will have a strong effect on the energetics of a cooling flow and perhaps will shut off mass dropout. The main problem with this idea is that the gas in a cluster is likely to be magnetized, and conventional wisdom says that magnetic fields severely suppress conduction relative to the Spitzer level. This is the topic of the present Letter.

We discuss in $\S 2.1$ the theory of conduction in a tangled magnetic field as developed by Rechester \& Rosenbluth (1978, RR) and Chandran \& Cowley (1998, CC); the theory predicts that the coefficient of thermal conduction is a factor $\sim 100-1000$ lower than the Spitzer coefficient. We then present in $\S 2.2$ and $\S 2.3$ an extension of the theory to a turbulent medium; we show that if turbulence extends over a factor of 100 or more in length scale, thermal conduction is almost as efficient as in Spitzer's theory. We conclude with a brief discussion in $\S 3$.

\section{THEORY OF THERMAL CONDUCTION IN A WEAKLY COLLISIONAL MAGNETIZED GAS}

\subsection{Conduction in a Chaotic Magnetic Field With a Single Scale}

In the presence of an ordered magnetic field, conduction is anisotropic. Electrons stream freely parallel to the field line, so the parallel diffusion constant is almost equal to the Spitzer value: $\kappa_{\|} \sim \kappa_{\mathrm{Sp}} / 3$. (The factor of

\footnotetext{
${ }^{1}$ Institute for Advanced Study, Princeton, NJ 08540; Permanent address: Harvard-Smithsonian Center for Astrophysics, Cambridge, MA 02138; rnarayan@cfa.harvard.edu

2 Canadian Institute for Theoretical Astrophysics, Toronto, ON, M5S 3H8, Canada; medvedev@cita.utoronto.ca;
} http://www.cita.utoronto.ca/ medvedev/

${ }^{3}$ The coefficient $\kappa_{c}$ which enters in the heat flux equation, $q=-\kappa_{c} \nabla T$, is related to $\kappa_{\mathrm{Sp}}$ by $\kappa_{c}=n k_{B} \kappa_{\mathrm{Sp}}$, where $k_{B}$ is the Boltzmann constant. Note also that heat diffusion and particle diffusion have slightly different coefficients, differing by a factor of order unity. 
$1 / 3$ is because diffusion is in one dimension rather than three, see CC). Perpendicular to the field, however, electrons follow circular Larmor orbits with radius $\rho_{e} \ll \lambda$. Since an electron moves only a distance $\sim \rho_{e}$ in each scattering, the perpendicular diffusion constant is given by $\kappa_{\perp} \sim \rho_{e}^{2} / t_{\text {Coul }} \sim\left(\rho_{e} / \lambda\right)^{2} \kappa_{\mathrm{Sp}} \ll \kappa_{\mathrm{Sp}}$. For a galaxy cluster with a magnetic field of $\sim 10^{-6} \mathrm{G}$, we have $\rho_{e} \sim 10^{-12} \lambda$, and $\kappa_{\perp}$ is effectively zero.

Thermal conduction behaves very differently when the magnetic field is chaotic. The theory for a tangled field with a single coherence length $l_{B}$ was developed by RR and has been recently revived in the astrophysical context by CC. Since the field is chaotic, the separation $r$ of two nearby field lines must have a Lyapunov-like scaling as a function of distance $l$ along the field:

$$
r \sim r_{0} \exp \left(l / L_{\text {Lyap }}\right),
$$

where $r_{0}$ is the initial separation of the two lines. As there is only one characteristic scale in the problem, namely $l_{B}$, we expect $L_{\text {Lyap }} \sim l_{B}$.

Following RR and $\mathrm{CC}$, let us consider the evolution of a compact cloud of electrons of initial size $\rho_{e}$. With time, the electrons diffuse parallel to the field, with a diffusion constant $\kappa_{\|}$. As the electron cloud spreads out, its perpendicular extent diverges exponentially according to equation (1). Thus, by the time the electrons have diffused a Rechester-Rosenbluth distance $L_{R R} \sim l_{B} \ln \left(l_{B} / \rho_{e}\right) \sim$ $30 l_{B}$ along the field line, their transverse separation is of order $l_{B}$. The numerical coefficient 30 corresponds to $l_{B} / \rho_{e} \sim 10^{13}$, a typical value for a galaxy cluster (assuming $l_{B}$ is a fraction of the radius). Being a logarithmic factor, the numerical value is insensitive to details. When electrons have moved a distance $L_{R R}$ along the tangled field line, their three-dimensional root mean square (rms) displacement is $R_{*}$, where $R_{*}^{2} \sim L_{R R} l_{B} \sim 30 l_{B}^{2}$. Beyond $R_{*}$, the motion of an electron is isotropic and uncorrelated with its previous path.

Let us define $t_{*}$ as the time it takes for electrons to diffuse a distance $L_{R R}$ along the field: $t_{*} \sim L_{R R}^{2} / \kappa_{\|}$. For $t<t_{*}$, electrons diffuse anisotropically: $l \sim\left(\kappa_{\|} t\right)^{1 / 2}, r \sim$ $\rho_{e} \exp \left[\left(\kappa_{\|} t\right)^{1 / 2} / l_{B}\right]$. For $t>t_{*}$, however, electrons diffuse isotropically, and move in three dimensions according to $R \sim\left(\kappa_{*} t\right)^{1 / 2}$, where

$$
\kappa_{*} \sim R_{*}^{2} / t_{*} \sim\left(l_{B} / L_{R R}\right) \kappa_{\|} \sim 10^{-2} \kappa_{\mathrm{Sp}} .
$$

We see that for $R>R_{*}$ the conduction is many orders of magnitude more efficient than when the field is ordered. However, $\kappa_{*}$ is still a factor $\sim 100$ less than $\kappa_{\mathrm{Sp}}$. The conduction time is correspondingly $\sim 100$ times longer than the Spitzer time $t_{\mathrm{Sp}}$. As Table 1 shows, such weak conduction is unlikely to have an important effect on cooling flows.

The estimate given in equation (2) is valid so long as $\lambda<l_{B}$. This condition, which is likely to be satisfied by the gas in clusters (compare $\lambda$ with $l_{B} \sim R /$ (few) in Table 1 ), ensures that collisions enable electrons to pass through magnetic mirrors caused by inhomogeneities in the field. If $\lambda>l_{B}$, conduction is suppressed by an additional factor $\theta<1$ (Chandran et al. 1999; Malyshkin \& Kulsrud 2001) since only a fraction of the electrons are able to penetrate the mirrors. This would cause the conduction time to increase by a factor $1 / \theta$.

\subsection{Conduction in a Multiscale Chaotic Magnetic Field}

A key assumption of the RR theory is the presence of a single Lyapunov length scale $L_{\text {Lyap }} \sim l_{B}$. However, if the medium is turbulent, chaotic fluctuations will be present over a wide range of length scales. We generalize the theory for such a multiscale medium.

We begin by re-expressing the single-scale theory as follows. When two field lines are separated by a distance $r$ smaller than $l_{B}$, their mean square separation $\left\langle r^{2}\right\rangle$ increases with distance $l$ along the field line according to a Lyapunov-like scaling. However, when $r>l_{B}$, the increase is given by the usual diffusion law, where $\left\langle r^{2}\right\rangle$ increases by $\Delta r^{2} \sim l_{B}^{2}$ for a parallel displacement $\Delta l \sim l_{B}$. Thus, we may describe the evolution in the two regimes, $\left\langle r^{2}\right\rangle<l_{B}^{2}$ and $\left\langle r^{2}\right\rangle>l_{B}^{2}$, by the following two differential equations:

$$
d\left\langle r^{2}\right\rangle / d l \sim 2\left\langle r^{2}\right\rangle / l_{B}, \quad d\left\langle r^{2}\right\rangle / d l \sim l_{B}^{2} / l_{B}=l_{B} .
$$

Consider now a tangled magnetic field with a range of scales, and assume that the statistics of the magnetic field fluctuations are described by the Goldreich \& Sridhar (1995, GS) theory of Alfvenic MHD turbulence. In a GS turbulent cascade, there is a range of scales $l_{\perp}$ perpendicular to the field, extending from a minimum scale $l_{\perp \text {, min }}$ to a maximum scale $l_{B}$. The fluctuations are anisotropic, so that for a given perpendicular scale $l_{\perp}$ the corresponding parallel coherence scale $l_{\|}$is given by

$$
\left(l_{\|} / l_{B}\right) \sim\left(l_{\perp} / l_{B}\right)^{\alpha}, \quad l_{\perp, \min }<l_{\perp}<l_{B} .
$$

For simplicity, we have selected the normalization such that on the outer scale $l_{B}$, the fluctuations are isotropic: $l_{\perp} \sim l_{\|} \sim l_{B}$. The index $\alpha$ is equal to $2 / 3$ for strong MHD turbulence (GS) and 3/4 for intermediate turbulence (Goldreich \& Sridhar 1997).

Using the single-scale equations (3) as a guide, it is straightforward to write down corresponding equations for a medium with a spectrum of fluctuations. We then identify three regimes for the evolution of $\left\langle r^{2}\right\rangle$.

First, when $r \equiv\left\langle r^{2}\right\rangle^{1 / 2}<l_{\perp, \text { min }}$, all the fluctuation scales in the medium contribute to Lyapunov-like growth (assuming they all behave chaotically), so

$$
\frac{d\left\langle r^{2}\right\rangle}{d l} \sim 2\left\langle r^{2}\right\rangle \int_{1 / l_{B}}^{1 / l_{\perp, \text { min }}} \frac{d \ln k_{\perp}}{l_{\|}} \sim \frac{2\left\langle r^{2}\right\rangle}{l_{\|, \min }},
$$

where we have written $k_{\perp}=1 / l_{\perp}$, and we have ignored constants of order unity in the normalization of the integral. We see that the effective Lyapunov scale for the growth of $r$ is the parallel coherence length of the smallest scale fluctuations in the medium, i.e.

$$
r \sim r_{0} \exp \left(l / l_{\|, \min }\right), \quad l_{\|, \min } \sim l_{\perp, \min }^{\alpha} l_{B}^{1-\alpha} .
$$

Since usually $l_{\|, \min } \ll l_{B}$, the growth is rapid.

Once $r$ exceeds $l_{\perp, \min }$, the evolution switches to a second regime. We continue to have Lyapunov-like growth from scales $l_{\perp}>r$, but there is diffusion-like growth for scales $l_{\perp}<r$. The evolution equation for $l_{\perp, \min }^{2}\left\langle\left\langle r^{2}\right\rangle\left\langle l_{B}^{2}\right.\right.$ thus becomes

$$
\frac{d\left\langle r^{2}\right\rangle}{d l} \sim 2\left\langle r^{2}\right\rangle \int_{1 / l_{B}}^{1 / r} \frac{d \ln k_{\perp}}{l_{\|}}+\int_{1 / r}^{1 / l_{\perp, \min }} d \ln k_{\perp} \frac{l_{\perp}^{2}}{l_{\|}} .
$$


If $l_{B} \gg r \gg l_{\perp, \text { min }}$, each integral in equation (7) is dominated by the scale $r$. Substituting equation (4) in equation (7), we then obtain the solution

$$
r / l_{B} \sim\left(l / l_{B}\right)^{1 / \alpha}
$$

Remarkably, the separation between two neighboring field lines becomes of order $l_{B}$ for a parallel translation of only $\sim l_{B}$; this is much faster than in the RR theory which requires a parallel translation $\sim 30 l_{B}$. The solution $r \propto l^{1 / \alpha}$ corresponds exactly to $l_{\perp} \propto l_{\|}^{1 / \alpha}$ in the turbulence model (4). Thus, the rms separation of field lines grows along the "Goldreich-Sridhar cone."

When $r>l_{B}$, we enter a third regime, which corresponds to isotropic diffusion.

¿From equation (8), it is clear that thermal conduction in a multiscale chaotic field is almost as efficient as in Spitzer's theory. Replacing $L_{R R}$ by $l_{B}$ in equation (2), we estimate $\kappa_{\text {turb }} \sim \kappa_{\mathrm{Sp}} / 3$ (but see $\S 2.3$ for a better estimat p of the coefficient). As in the previous subsection, we ha assmed $\lambda<l_{B}$ and hat inelued a mag netic mirror factor $\theta$. In GS turbulence, perturbations on length scales $l_{\perp}<l_{B}$ have weak magnetic field fluctuations, $\triangle B / B, l_{\perp} / l_{\|} \sim\left(l_{\perp} / l_{B}\right)^{1-\alpha}<1$, and eause negligite mirroring. Only on the $l_{B}$ cause strong minroning, but these have a negligible effect so long as $\lambda<l_{B}$ Chandran et al. 1999; Aalyshlkin \& Kulsr id 2001).

\subsection{Numerical Solutions}

We have numerically integrated the differential equations (5) and (7), starting with an initial separation $r=$ $10^{-13} l_{B} \sim \rho_{e}$, and assuming $\alpha=2 / 3$ as appropriate for strong turbulence in the GS model. Figure 1 shows four numerical solutions for the evolution of $r$ as a function of distance $l$ along the field line, corresponding to four choices of the minimum scale $l_{\perp \text {, min }}$ of the turbulence. We see exponential growth of $r$ for $l<l_{\perp \text {,min }}$, and power-law growth for larger separations, confirming the scalings given in (6) and (8).

Let us define the decorrelation length $L_{\text {dec }}$ as the distance along the field line for which the transverse separation $r$ becomes equal to $l_{B}$. Figure 2 shows how $L_{\text {dec }}$ depends on $l_{\perp \text {, min }}$. When $l_{\perp \text {, min }} \sim l_{B}$, the turbulence is dominated by a single (outer) scale. This corresponds to the RR theory, and in this limit the decorrelation length is large, as expected. However, for $l_{\perp, \min } \lesssim 10^{-2} l_{B}$, we find that $L_{\text {dec }}$ is quite small, asymptoting to $\sim 1.6 l_{B}$. Since $L_{\mathrm{dec}}$ is the analog of the Rechester-Rosenbluth length $L_{R R}$ for a multiscale medium, we may replace $L_{R R}$ by $L_{\mathrm{dec}}$ in equation (2) to estimate the diffusion constant in a turbulent medium 4

$$
\kappa_{\text {turb }} \sim\left(l_{B} / L_{\text {dec }}\right) \kappa_{\|} \sim \kappa_{\mathrm{Sp}} / 5, \quad t_{\text {turb }} \sim 5 t_{\mathrm{Sp}} .
$$

Thus, if turbulence extends over at least two decades in scale, conduction is very efficient and approaches the Spitzer level to within a factor of a few.

\section{DISCUSSION}

Thermal conductivity in a homogeneous magnetic field is known to be highly anisotropic - it is Spitzer along the field, but extraordinarily reduced in the transverse direction. RR came up with the important insight that when the magnetic field is tangled and chaotic, thermal conduction is enhanced significantly by the exponential divergence of neighboring field lines. However, even with this effect, $\mathrm{CC}$ estimated that the conductivity in galaxy clusters is below Spitzer by a factor $\gtrsim 100$.

We have shown in this Letter that if the field is chaotic over a wide range of length scales (factor of 100 or more), as might happen with MHD turbulence (GS), thermal conduction is boosted to within a factor $\sim 5$ of the Spitzer value. Such strong conduction will have a significant effect on galaxy clusters (compare columns 6 and 8 in Table 1). It can transport heat to the center of a cluster to replace the energy lost through cooling, and it can also eliminate any thermal instability in the cooling gas. Thus, it may well reduce the need for large-scale mass dropout in cooling flows (Tucker \& Rosner 1983; Bertschinger \& Meiksin 1986). Only in the inner regions of some clusters (e.g. 3C295, Table 1) might there be significant dropout. It is worth noting that some authors have discussed potential problems with invoking such strong conduction Binney \& Cowie 1981; Bregman \& David 1988), while others have rebutted these arguments (Rosner \& Tucker 1989; Santos 2001).

An important requirement for the validity of our analysis is that the magnetic field should behave chaotically, i.e., it should exhibit Lyapunov-like behavior over a wide range of scales. Weak MHD turbulence consists of a superposition of Alfvén waves and is not chaotic. However, the model of strong and intermediate MHD turbulence developed by GS is chaotic, as indicated by the breakdown of perturbation theory (Goldreich \& Sridhar 1997).

It should be noted that our theory of thermal conduction does not require ongoing dynamic turbulence. Each episode of dynamic turbulence will leave behind a substantial level of frozen-in tangled fields even after the turbulent motions have ceased. Such leftover tangled fields should be sufficient to enhance conduction to the levels we estimate.

As a final point, we should discuss a serious caveat. Chandra has found evidence for sharp temperature jumps in a few clusters, e.g., Abell 2142 (Markevitch et al. 2000) and Abell 3667 (Vikhlinin et al. 2001). The observations indicate that conduction across the temperature jumps is far below Spitzer (Markevitch et al. 2000; Ettori \& Fabian 2000 , in apparent contradiction with the estimate presented here. Vikhlinin et al. (2001) propose that the magnetic field is stretched parallel to the interface, thus inhibiting diffusion across the field on small scales. Extending this idea, we suggest that when two distinct objects merge, as appears to be the case with the above clusters, the two regions (each of which is internally chaotic and highly conducting) may be thermally isolated from each other because their magnetic fields have not yet interpenetrated each other. It is unclear how long such magnetic isolation will survive.

The authors thank Stas Boldyrev and Andrei Gruzinov

\footnotetext{
${ }^{4}$ Since eqs. (5), (7) are approximate and may contain numerical coefficients of order unity multiplying the integrals, the numerical factor of $1 / 5$ in eq. (9) is approximate as well.
} 
for useful discussions and the referee for helpful comments. RN was supported in part by the W.M. Keck Foundation as a Keck Visiting Professor at the Institute for Advanced
Study. This work was supported in part by NSF grant AST-9820686.

\section{REFERENCES}

Allen, S. W., et al. 2001, MNRAS, 324, 842

Bertschinger, E., \& Meiksin, A. 1986, ApJ, 306, L1

Binney, J., \& Cowie, L. L. 1981, ApJ, 247, 464

Böhringer, H., et al. 2001, A\&A, 365, L181

Bregman, J. N., \& David, L. P. 1988, ApJ, 326, 639

Chandran, B. D. G., \& Cowley, S. C. 1998, Phys. Rev. Lett., 80, 3077 (CC)

Chandran, B. D. G., Cowley, S. C., Ivanushkina, M., \& Sydora, R. 1999, ApJ, 525, 638

Cowie, L. L., \& McKee, C. F. 1977, ApJ, 211, 135

David, L. P., et al. 2001, ApJ, 557, 546

Ettori, S. \& Fabian, A. C. 2000, MNRAS, 317, L57

Fabian, A. C. 1994, ARA\&A, 32, 277

Fabian, A. C., Mushotzky, R. F., Nulsen, P. E. J., \& Peterson, J. R. 2001, MNRAS, 321, L20

Gaetz, T. J. 1989, ApJ, 345, 666

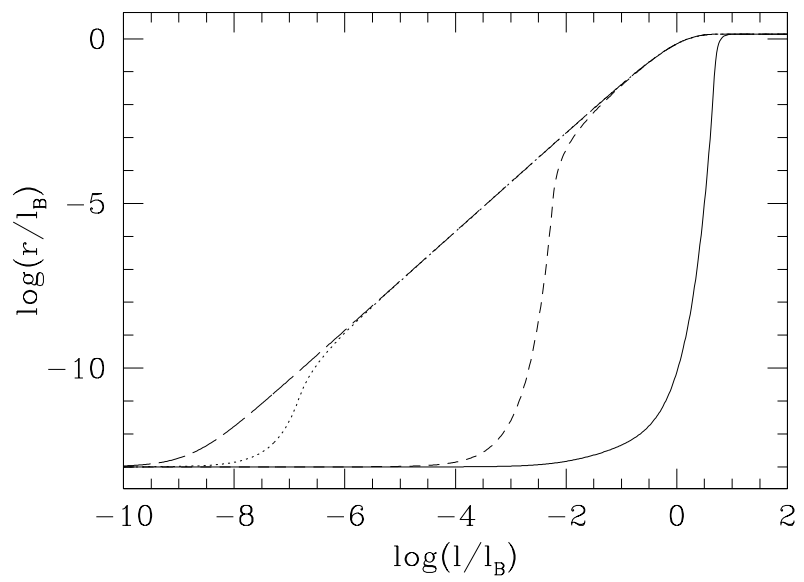

Goldreich, P., \& Sridhar, S. 1995, ApJ, 438, 763 (GS)

Goldreich, P., \& Sridhar, S. 1997, ApJ, 485, 680

Malyshkin, L., \& Kulsrud, R. 2001, ApJ, 549, 402

Markevitch, M., et al. 2000, ApJ, 541, 542

Molendi, S., \& Pizzolato, F. 2001, ApJ, 560, 194

Pistinner, S., \& Shaviv, G. 1996, ApJ, 459, 147

Rechester, A. B., \& Rosenbluth, M. N. 1978, Phys. Rev. Lett., 40, 38 (RR)

Rosner, R., \& Tucker, W. H. 1989, ApJ, 338, 761

Santos, S. D. 2001, MNRAS, 323, 930

Sarazin, C. L. 1988, X-ray emission from clusters of galaxies (Cambridge: Cambridge University Press)

Spitzer, L. 1962, Physics of fully ionized gases (New York: Interscience)

Tucker, W. H., \& Rosner, R. 1983, ApJ, 267, 547

Vikhlinin, A., Markevitch, M., \& Murray, S.S. 2001, ApJ, 551, 160

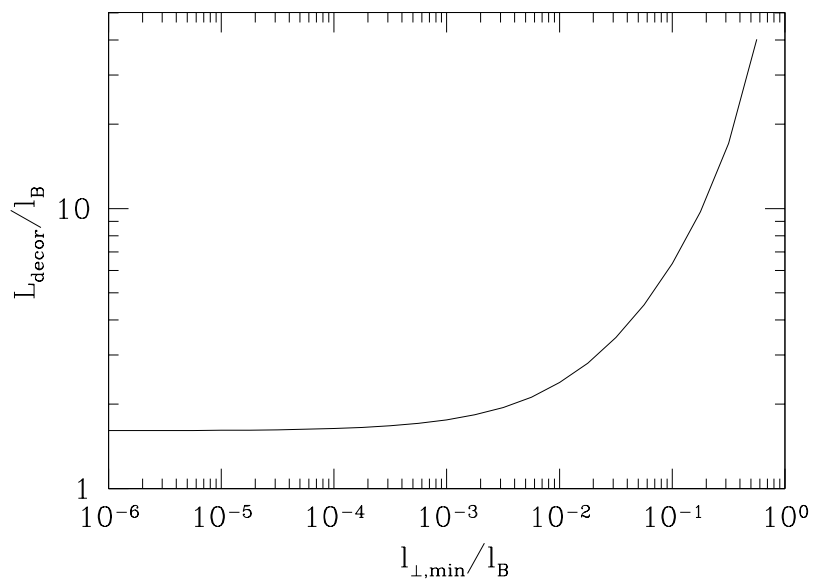

FIG. 1.- Variation of the rms transverse displacement $r$ of two field lines vs longitudinal distance $l$ along the field lines, for $\alpha=2 / 3$.

Four choices of the minimum scale of turbulence are shown, $l_{\perp, \min } / l_{B}=10^{-1}$ (solid line), $10^{-5}$ (dashed), $10^{-11}\left(\sim \rho_{p} / l_{B}\right.$, dotted), $10^{-13}$ $\left(\sim \rho_{e} / l_{B}\right.$, long-dashed), which correspond to $l_{\|, \min } / l_{B}=2.2 \times 10^{-1}, 4.6 \times 10^{-4}, 4.6 \times 10^{-8}, 2.2 \times 10^{-9}$, respectively.

FIG. 2.- Variation of the decorrelation length $L_{\mathrm{dec}}$ vs $l_{\perp, \min }$. Note that $L_{\mathrm{dec}} \lesssim 2 l_{B}$ for $l_{\perp, \min } \lesssim 10^{-2} l_{B}$.

TABLE 1

Comparison of the Cooling Time and the Conduction Time in Hydra A And 3C295

\begin{tabular}{cccccccc}
\hline \hline $\begin{array}{c}\text { Cluster } \\
\text { name }\end{array}$ & $\begin{array}{c}R \\
(\mathrm{kpc})\end{array}$ & $\begin{array}{c}n \\
\left(\mathrm{~cm}^{-3}\right)\end{array}$ & $\begin{array}{c}k T \\
(\mathrm{keV})\end{array}$ & $\begin{array}{c}\lambda \\
(\mathrm{kpc})\end{array}$ & $\begin{array}{c}t_{\text {cool }} \\
(\mathrm{Gyr})\end{array}$ & $\begin{array}{c}t_{\mathrm{Sp}} \\
(\mathrm{Gyr})\end{array}$ & $\begin{array}{c}t_{\text {turb }} \\
(\mathrm{Gyr})\end{array}$ \\
\hline $\begin{array}{c}\text { Hydra A } \\
\text { " }\end{array}$ & 100 & 0.005 & 3.6 & 0.8 & 5 & 0.5 & 2 \\
3C295 & 10 & 0.06 & 3.1 & 0.05 & 0.5 & 0.09 & 0.4 \\
"1 & 10 & 0.008 & 5.0 & 0.9 & 7 & 0.3 & 2 \\
& & 0.15 & 3.0 & 0.02 & 0.3 & 0.2 & 1 \\
\hline
\end{tabular}

